\title{
Heart failure due to cytomegalovirus myocarditis in immunocompetent young adults: a case report
}

\author{
Moacyr Magno Palmeira1,2,4*, Hellen Yuki Umemura Ribeiro², Yan Garcia Lira², \\ Fernando Octávio Machado Jucá Neto² Ivone Aline da Silva Rodrigues², Letícia Nazareth Fernandes da Paz ${ }^{2}$ \\ and Maria da Conceição Nascimento Pinheiro ${ }^{3}$
}

\begin{abstract}
Background: Cardiac complications constitute a rare clinical manifestation of cytomegalovirus (CMV) infection. This virus is usually asymptomatic in immunocompetent individuals. We report a case of myocarditis and cardiac insufficiency due to primary CMV infection. Serological tests by using ELISA method showed positive results for the virus.

Case presentation: A 41-year-old man with no prior comorbidities presenting with dyspnoea, fever, and oedema was admitted to the cardiac emergency service. He had fever and dry cough, which aggravated into progressive respiratory distress, lower limb oedema, and orthopnoea 30 days prior to hospitalisation. The electrocardiogram revealed sinus tachycardia, first-degree right bundle branch block, and ventricular and left atrial overload as well as diffuse and nonspecific disturbances of ventricular repolarization. Serological tests were conducted, and $\operatorname{lgM}(1.54 \mathrm{UI} / \mathrm{mL})$ and $\operatorname{lgG}(2.5 \mathrm{UI} / \mathrm{mL})$ were found positive only for CMV by using ELISA. The patient was diagnosed with cardiac insufficiency due to CMV myocarditis. He was treated with ganciclovir for 10 days and received supportive medication.
\end{abstract}

Conclusion: This case reaffirms the possibility of cardiac involvement in CMV infection and emphasises the importance of viral aetiologies as differential diagnoses for acute myocarditis.

Keywords: Cytomegalovirus infection, Myocarditis, Heart disease

\section{Background}

Cytomegalovirus (CMV) infection is a prevalent infectious disease worldwide; however, its prevalence varies with geographical regions and socioeconomic statuses [1]. CMV belongs to the Herpesviridae family and Betaherpesvirinae subfamily type 5 ; it is a DNA virus with the ability of remaining latent for a long time [2]. Its prevalence ranges from 30 to $100 \%$ in the general population with prior exposure to the virus as determined by serological tests. This prevalence is inversely proportional to socioeconomic status and directly proportional to age [3]. Adults and adolescents, such as day-care workers and school teachers, are at higher risk for acute CMV

*Correspondence: palmeiramm@ig.com.br

${ }^{4} 60$ Amazonas square, Jurunas, 66025-070 Belém, PA, Brazil

Full list of author information is available at the end of the article infection because of the close contact with children who may be infected [1]. Primary infection is usually an asymptomatic event in immunocompetent individuals. However, there are clinical presentations that are described as classic influenza-like syndrome or mononucleosis-like syndrome with symptoms of the classic fever, pharyngitis, and lymphadenopathy triad $[1,5]$. The intensity and clinical symptoms vary and include systemic disorders such as hepatitis, hematologic diseases, or meningoencephalitis; additionally, it can be associated with high mortality rates $[2,4,5]$.

Cardiovascular complications constitute a rare manifestation of CMV infection; it is a diagnostic challenge in the emergency cases [4]. We describe a previously healthy man who developed myocarditis and cardiac insufficiency due to primary CMV infection. 


\section{Case presentation}

A 41-year-old man, unemployed, brown, parent of two school-age children, with no prior comorbidities, and presenting with dyspnoea, fever, and oedema was admitted to the cardiac emergency service. The patient had undergone no related interventions in the past and had no cardiac risks based on his family history.

He had fever and dry cough, which aggravated into progressive respiratory distress, lower limb oedema, and orthopnoea 30 days prior to hospitalisation. Physical examination revealed fever $\left(38{ }^{\circ} \mathrm{C}\right)$, pallor $(++/ 4+)$, dyspnoea, tachycardia [heart rate $(\mathrm{HR})=136 \mathrm{bpm}$ ], cardiac gallop rhythm (presence of S3), mitral systolic murmur as well as absent breath sounds at the lung base, and crackles and rales up to the middle third of both the lungs. Additionally, he experienced hepatomegaly, splenomegaly, painless oedema, positive pitting oedema $(++++/ 4+)$ of the lower limbs, bruises, and petechiae (Fig. 1).

The electrocardiogram revealed sinus tachycardia, firstdegree right bundle branch block, and ventricular and left atrial overload as well as diffuse and nonspecific disturbances of ventricular repolarization (Fig. 2). The chest radiographs revealed bilateral pleural effusion in the lung base and an enlarged cardiac silhouette (Fig. 3). A transthoracic echocardiogram revealed $35 \%$ ejection fraction (EF) of the left ventricle (LV) and an increase in the left cavity $(70 \mathrm{~mm} \mathrm{LV})$. Coronary angiography revealed no obstructive atherosclerosis lesions in the coronary artery.

Serological tests were conducted for HIV-1 and HIV2, hepatitis B and C, CMV, and Chagas disease. The

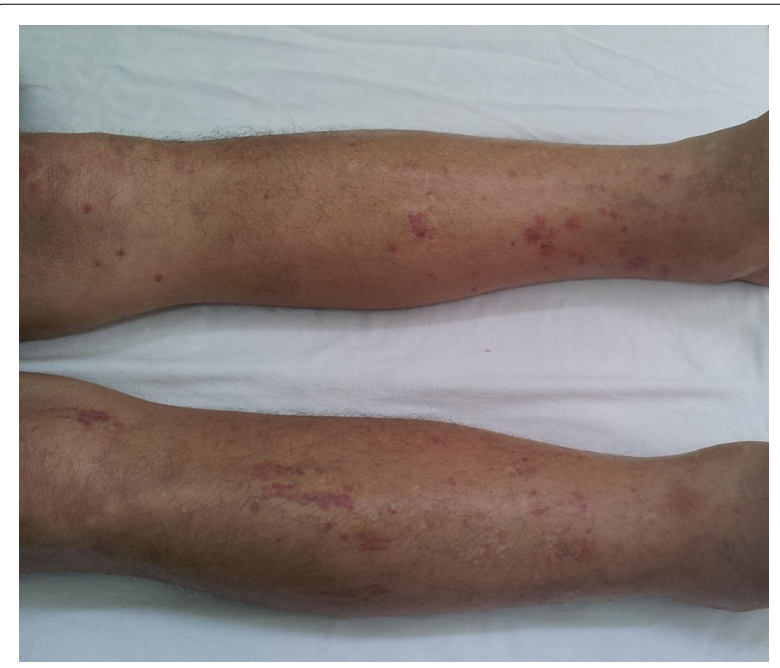

Fig. 1 Patient's lower limbs presenting with severe oedema, ecchymosis, and petechiae. Patient presented with painless oedema, positive pitting oedema $(++++/ 4+)$ of the lower limbs, ecchymosis, and petechiae serological tests results were negative for all the viruses except CMV, which included positive results for IgM $(1.54 \mathrm{UI} / \mathrm{mL})$ and $\operatorname{IgG}(2.5 \mathrm{UI} / \mathrm{mL})$ by using ELISA.

Laboratory-based tests conducted upon admission revealed the following: haemoglobin level, $9.15 \mathrm{~g} / \mathrm{dL}$; leukocyte number, $8400 / \mathrm{mm}^{3}$; segmented neutrophil fraction, $77.5 \%$; platelet number, $289,000 / \mathrm{mm}^{3}$; unchanged coagulation; erythrocyte sedimentation rate, $120 \mathrm{~mm} / \mathrm{h}$; and C-reactive protein (CRP) level, $96 \mathrm{mg} / \mathrm{dL}$. Troponin I level increased but the creatinine kinase-MB level did not increase (3.12 and $2.1 \mathrm{ng} / \mathrm{mL}$, respectively). The renal scores and ionogram values were within the reference values. In addition, the patient showed significantly increased levels of canalicular liver enzymes [alkaline phosphatase (ALP) and gamma glutamyl transferase (GGT)] during hospitalisation.

The patient was diagnosed with cardiac insufficiency due to CMV myocarditis. He was administered $250 \mathrm{mg}$ of ganciclovir IV twice a day for 10 days and received supportive medication that included oral administration of $0.25 \mathrm{mg}$ of digoxin once a day, $50 \mathrm{mg}$ of losartan twice a day, $25 \mathrm{mg}$ of carvedilol twice a day, $40 \mathrm{mg}$ of furosemide twice a day, and $25 \mathrm{mg}$ of spironolactone once a day. After receiving the anti-viral medication, the patient's liver enzyme levels decreased, as revealed by laboratorybased tests (Fig. 4); in addition, the hepatosplenomegaly improved. Abdominal CT was performed following the drug therapy, and normal liver characteristics were revealed with no biliary tract dilatation. The ecchymotic spots and petechiae that were observed upon admission and that had emerged during the course of infection were also treated.

The patient showed improvement in clinical and laboratory-based tests and was discharged in functional class I (New York Heart Association) after 14 days of hospitalisation. The serological tests confirmed CMV infection (IgM, $0.9 \mathrm{UI} / \mathrm{mL}$ and IgG, $10.1 \mathrm{UI} / \mathrm{mL}$ ). One month after discharge, routine echocardiography revealed a $75 \%$ EF of the LV and almost normal values for the diameter of the left chambers. The cardiac enzyme levels were normal.

\section{Discussion}

Generally, in immunocompetent adults, CMV infection is either asymptomatic or involves a typical disease course similar to a self-limited mononucleosis-like syndrome. The infection rarely causes severe complications of specific organs; however, acute gastrointestinal, cardiovascular, neurological, and/or liver disorders have been reported [1].

Evidence of cardiac events, particularly acute pericarditis or myocarditis due to CMV infection, is uncommon in immunocompetent patients without any associated risk 


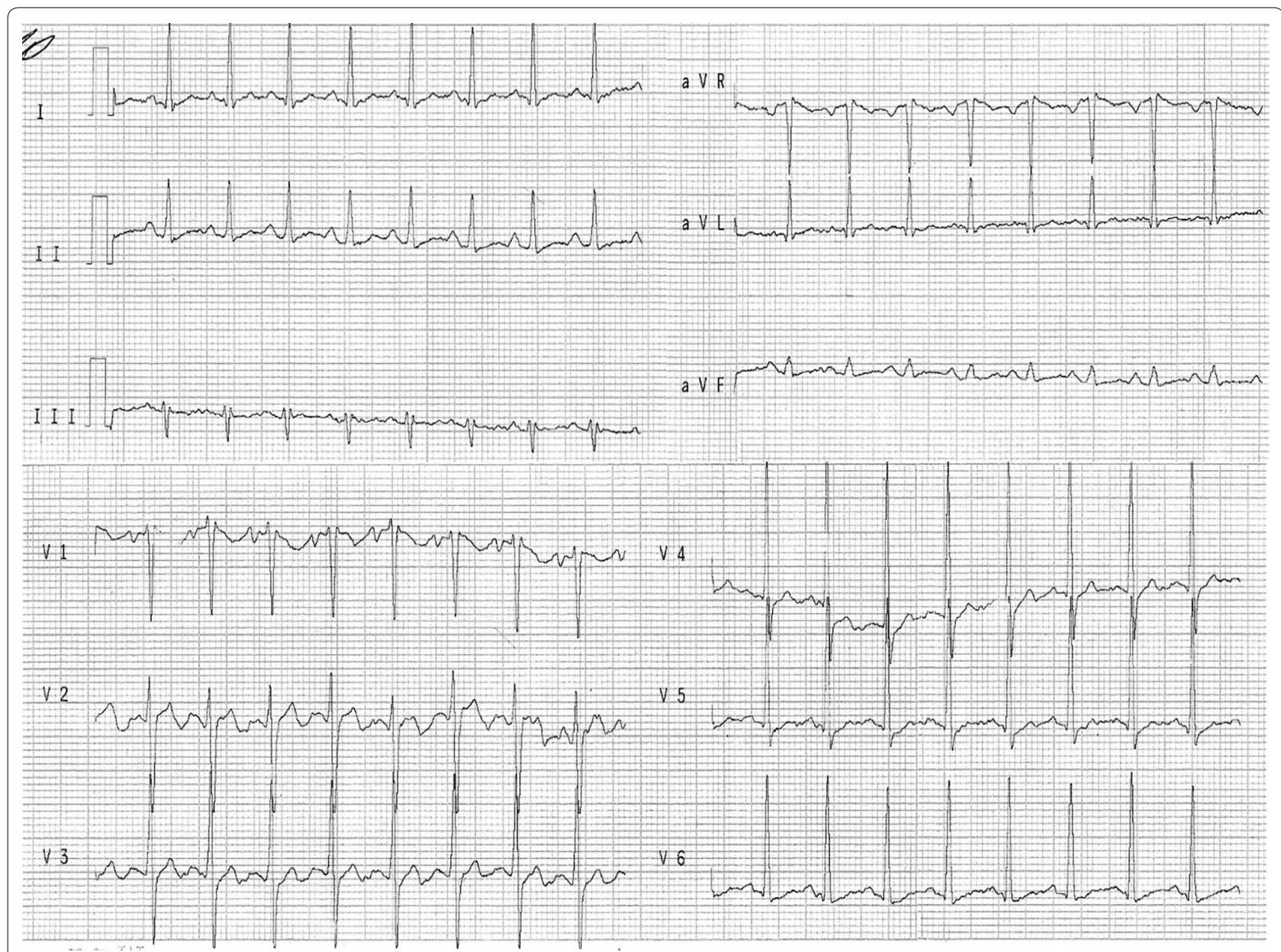

Fig. 2 Electrocardiogram revealing sinus tachycardia, first-degree right bundle branch block, and other cardiovascular disorders. The electrocardiogram reveals sinus tachycardia, first-degree right bundle branch block, and ventricular and left atrial overload, as well as diffuse and nonspecific disturbances of ventricular repolarization

factor [4]. Normally, these changes occur asymptomatically and, in most cases, are diagnosed as incidental findings during the typical course of mononucleosis-like syndrome $[4,5]$. However, there may occasionally be serious complications associated with infection, such as cardiogenic shock, tamponed, or acute cardiac insufficiency [5].

The diagnosis of myocarditis is a constant challenge in medical practice because of high clinical variability and high risk of sudden death, which can progress to dilated cardiomyopathy in approximately $10 \%$ of these patients [6]. Myocarditis is defined as inflammation of the heart muscle and may involve the myocytes, interstitium, vascular elements, and pericardium [6]. Infectious agents, especially viral agents, are the most important etiologic agents [7].

Owing to their risks and high costs, use of endomyocardial biopsies for making diagnoses remains controversial. The incremental diagnostic, prognostic, and therapeutic values of risks associated with such an invasive procedure must be evaluated. Particularly, such an evaluation must be conducted when the aetiology of myocarditis, based on positive results of CMV serological tests, is very strongly suspected. An excellent clinical outcome after ganciclovir therapy that included $>100$ \% EF increase confirmed the initial diagnosis made by using a non-invasive test [8].

Enteroviruses are widely associated with myocarditis and dilated cardiomyopathy, and CMV has sporadically been implicated in this process. In a survey of etiologic agents in cases of ventricular dysfunction designated as "idiopathic," expression of the CMV genome was detected in only $0.8 \%$ of the cases [7].

In a study based on myocardial autopsy, the CMV DNA was found in $38 \%$ of the patients with myocarditis as 


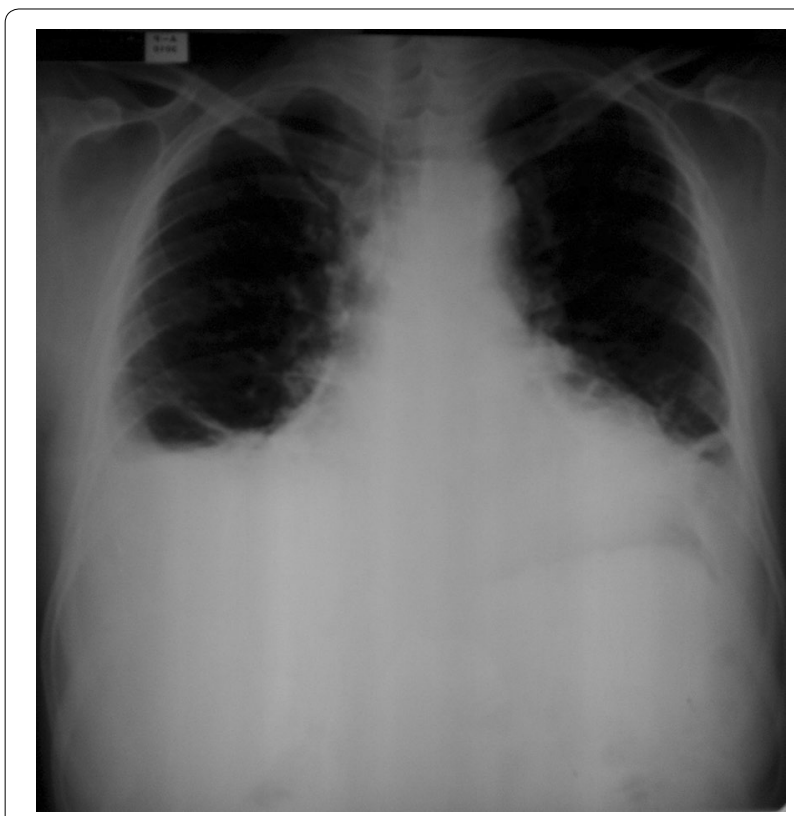

Fig. 3 Chest radiograph showing cardiomegaly and bilateral pleural effusions in the lung bases. The chest radiographs reveal bilateral pleural effusion in the lung base and an enlarged cardiac silhouette

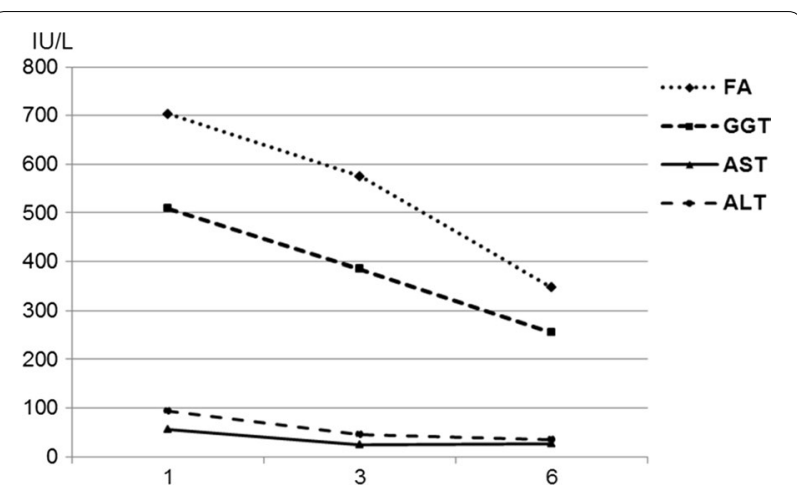

Fig. 4 Laboratory tests-based evolution of liver enzymes during ganciclovir therapy. After receiving the anti-viral medication, the patient exhibited a regression in his liver enzyme levels based on laboratory tests; changes are shown from day 1 to day 6 of the therapy

the death cause; in contrast, no CMV DNA was found in the control subjects. The authors suggested that an antiviral therapy could be potentially effective for treating CMV infection in patients with severe acute myocarditis; our study findings are similar to that of this study. We observed an excellent clinical outcome after ganciclovir therapy [9].

The pathophysiology of viral myocarditis involves a complex process characterized by three distinct phases: infection of myocytes, production of toxins, and immune-mediated cytotoxicity. Thus, injury occurs because of the direct cytotoxic effect of the causative agent, the secondary immune response caused by the infectious agent, cytokine expression in the myocardium, and the aberrant induction of apoptosis. It is believed that the progression to dilation and contractile dysfunction occurs in a linear manner and that the manifestation of symptoms may occur at any of the three phases [6].

The prognosis appears to be favourable in patients who survive the initial critical phase. Certain studies have shown a 50-80\% chance of resolving dilated cardiomyopathy within the first 2 years of the clinical onset [6]. In the present case, satisfactory progress was seen that included the recovery of ventricular function and decreased dilation of the cardiac chambers.

Abnormalities based on liver function tests are often reported in immunocompetent adults with clinically significant primary CMV infection. The blood levels of transaminases frequently increase slightly or moderately; however, the levels rarely exceed five times the reference values; in addition, the alanine transaminase (ALT) levels are more affected than the aspartate transaminase (AST) levels are. Increase in ALP, GGT, and total bilirubin levels are uncommon [1].

In this case, minimal change was detected in the AST and ALT levels, whereas the GGT and ALP levels increased significantly, which was expected during viral infection. These findings led to a hypothesis of asymptomatic viral cholangitis with a self-limited course due to anti-viral therapy. These enzymes decreased during therapy, and the abdominal ultrasound examination performed during the patient follow-up revealed no signs of gallstones or liver changes.

Additionally, petechiae and ecchymotic spots were observed on the lower limbs, which had appeared during the course of infection and disappeared a few days after the completion of the drug therapy. This trend suggested the possible evolution of infectious purpura fulminans caused by CMV infection. Vascular involvement by CMV has been reported; this virus induces vascular changes and triggers a cascade of events that can generate perivascular inflammation and even thrombosis [10].

The role of anti-viral therapy in the management of visceral diseases caused by CMV in immunocompromised patients, particularly transplant recipients, has been well established. However, the use of these agents in immunocompetent individuals is not well established, and no definitive conclusion has been established regarding their indication [4]. Nevertheless, several studies indicate the benefits of using anti-viral therapy, particularly during the acute phase of viral infection and in severe cases [6].

In the present case, the acute nature of the viral infection and the severity of the clinical situation prompted us specifically to provide ganciclovir therapy. The outcome 
was favourable and included the resolution of symptoms and a marked improvement of the cardiac, respiratory, and liver manifestations after specific anti-viral therapy.

\section{Conclusions}

This case reaffirms the possibility of cardiac involvement in CMV infection and emphasises the importance of viral aetiologies as differential diagnoses for acute myocarditis.

\section{Abbreviations}

ALP: alkaline phosphatase; ALT: alanine transaminase; AST: aspartate transaminase; CMV: cytomegalovirus; CRP: C-reactive protein; CT: computerized tomography; EF: ejection fraction; ELISA: enzyme-linked immunosorbent assays; GGT: gamma glutamyl transferase; HIV: human immunodeficiency virus; HR: heart rate; IV: intravenous; LV: left ventricle.

\section{Authors' contributions}

MMP and MCNP conceived and designed the experiment. HYUR, YGL, FOMJN, $I A S R$, and LNFP participated in sample collection and performed the experiments. MMP, LNFP, and MCNP were involved in patient care. MMP, HYUR, YGL, FOMJN, IASR, LNFP, and MCNP drafted the manuscript and contributed to the transcription. MMP, HYUR, FOMJN, and MCNP contributed significantly in the critical revision of the manuscript. MMP, HYUR, YGL, FOMJN, IARS, LNFP, and MCNP designed and participated in the review. All authors read and approved the final manuscript.

\section{Author details}

${ }^{1}$ Section of Urgency and Emergency, Division of Cardiology, Department of Intensive Care, Fundação Pública Estadual Hospital de Clínicas Gaspar Vianna, 2000 Alferes Costa street, Pedreira, 66087-660 Belém, PA, Brazil.

2 Section of Urgency and Emergency, Division of Cardiology, Department of Clinical Care, Universidade do Estado do Pará, 2623 Perebebuí street, Marco, 66087-670 Belém, PA, Brazil. ${ }^{3}$ Section of Human Pathology, Division of Tropical Diseases, Department of Infectious Diseases, Universidade Federal do Pará, 92 Generalíssimo Deodoro avenue, 66055-240 Umarizal, PA, Brazil. ${ }^{4} 60$ Amazonas square, Jurunas, 66025-070 Belém, PA, Brazil.

\section{Acknowledgements}

This study did not receive any funding support. The authors would like to thank the medical staff of the hospital Fundação Pública Estadual Hospital de Clínicas Gaspar Vianna for their support in patient's care.

\section{Competing interests}

The authors declare that they have no competing interests and have received no specific funding for this work.

\section{Availability of data and materials}

All information is included in the manuscript and its supplementary files.

\section{Consent for publication}

A written informed consent was obtained from the patient for publication of this case report and any accompanying images.

\section{Ethics approval and consent to participate}

This work was approved by the ethics committee "Fundação Pública Estadual Hospital de Clínicas Gaspar Vianna".

Received: 12 August 2015 Accepted: 23 July 2016

Published online: 05 August 2016

\section{References}

1. Hurt C, Tammaro D. Diagnostic evaluation of mononucleosis-likeillnesses. Am J Med. 2007;120:911.

2. Kenneson A, Cannon MJ. Review and meta-analysis of the epidemiology of congenital cytomagalovirus (CMV) infection. Rev Med Virol. 2007:17(4):253-76. doi:10.1002/rmv.535.

3. Matos SB, Meyer R, Lima FWM. Seroprevalence and serum profile of cytomegalovirus infection among patients with hematologic disorders in Bahia State, Brazil. J Med Virol. 2011;83(2):298-304. doi:10.1002/jmv.21965.

4. Roubille C, Brunel AS, Gahide G, Kovacsik HV, Quellec A. Cytomegalovirus (CMV) and acute myocarditis in an immunocompetent patient. Intern Med. 2010;49(2):131-3. doi:10.2169/internalmedicine.49.2313.

5. Vanstechelman F, Vandekerckhove H. Cytomegalovirus myocarditis in an immunocompetent patient. Acta Cardiol. 2012;67:257-60.

6. Feldman AM, McNamara D. Myocarditis. N Eng J Med. 2000;343:1388-98.

7. Kuhl U, Pauschinger M, Noutsias M, Seeberg B, Bock T, Lassner D, et al. High prevalence of viral genomes and multiple viral infections in the myocardium of adults with "idiopathic" left ventricular dysfunction. Circulation. 2005:111(7):887-93. doi:10.1161/01.CIR.0000155616.07901.35.

8. Cooper LT, Baughman KL, Feldman AM, Frustaci A, Jessup M, Kuhl $U$, et al. The role of endomyocardial biopsy in the management of cardiovascular disease. Circulation. 2007;111(19):2216-33. doi:10.1161/ CIRCULATIONAHA.107.186093.

9. Kytö V, Vuorinen T, Saukko P, Lautenschlager I, Lignitz E, Saraste A, VoipioPulkki LM. Cytomegalovirus infection of the heart is common in patients with fatal myocarditis. Clin Infect Dis. 2005;40(5):683-8.

10. Ofotokun I, Carlson C, Gitlin SD, Elta G, Singleton TP, Markovitz DM. Acute cytomegalovirus infection complicated by vascular thrombosis: a case report. Clin Infect Dis. 2001;32:983-6.

\section{Submit your next manuscript to BioMed Central} and we will help you at every step:

- We accept pre-submission inquiries

- Our selector tool helps you to find the most relevant journal

- We provide round the clock customer support

- Convenient online submission

- Thorough peer review

- Inclusion in PubMed and all major indexing services

- Maximum visibility for your research

Submit your manuscript at www.biomedcentral.com/submit 